\title{
Localization of $\beta$-defensin genes in non human primates
}

\author{
M. Ventura, ${ }^{1}$ M. Boniotto, ${ }^{2}$ M. Pazienza, ${ }^{1}$ V. Palumbo, ${ }^{2}$ M.F. Cardone, ${ }^{1}$ M. Rocchi, ${ }^{1}$ A. Tossi, ${ }^{4}$ \\ A. Amoroso, ${ }^{2,3}$ S. Crovella ${ }^{2,3}$
}

${ }^{1}$ University of Bari, DAPEG Genetics Section, Bari; 'University of Trieste, Reproduction and Development

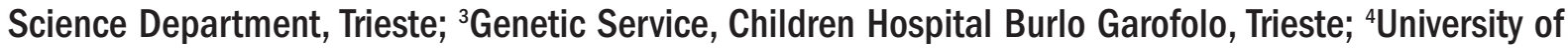

Trieste, Department of Biochemistry, Biophysics and Chemistry of Macromolecules, Trieste, Italy

(C2003, European Journal of Histochemistry

Defensins are a family of host defence peptides that play an important role in the innate immunity of mammalian and avian species. In humans, four $\beta$-defensins have been isolated so far, corresponding to the products of the genes DEFB1 (h-BD1, GenBank accession number NM_005218); DEFB4 (h-Bd2, NM_004942.2), DEFB103 (h-BD3, NM 018661); and DEFB104 (hBD4, NM 080389) mapping on chromosome 8p23.22. We have localized $\beta$ defensin genes on metaphasic chromosomes of great apes and several non-human primate species to determine their physical mapping. Using fluorescent in situ hybridization and BAC probes containing the four $\beta$-defensin genes, we have mapped the homologous regions to the $\beta$-defensin genes on chromosome 8p23-p.22 in non-human primates, while no signals were detected on prosimians chromosomes.

Key words: Innate immunity, molecular evolution, FISH, homology.

Correspondence: Sergio Crovella, Reproduction and Development Science Department, University of Trieste, via dell'Istria 65/1, 34137 Trieste, Italy.

Phone: international +39.040 .3785538 . Fax: international +39.040.3785540. E-mail: crovella@burlo.trieste.it

Paper accepted on January 26, 2004

European Journal of Histochemistry 2004; vol. 48 issue 2 [Apr-Jun]: 195-200
I $\mathrm{n}$ humans, four $\beta$-defensins have been isolated and well characterized so far: i) DEFBI (hBD1, GenBank accession number NM_005218) is constitutively expressed in kidney tubules and at lower levels in the pancreas, lungs and genitourinary tract (Bensch et al. 1995; Schonwetter et al. 1995; Diamond et al.1996; Zhao et al. 1996; Goldman et al. 1997); ii) DEFB4 (h$\mathrm{Bd} 2, \mathrm{NM}$ _004942.2) is primarily induced in skin and other epithelia during inflamation (Harder et al. 1997b; Hiratsuka et al. 1998; Liu et al. 1998; Mathews et al. 1999); iii) DEFB103 (h-BD3, NM_018661) is secreted by keratinocytes and epithelial cells of the respiratory tract after inflammatory stimuli or after contact with Gram-negative or Gram-positive bacteria (Harder et al. 1997a) and iv) DEFB104 (hBD4, NM_080389), whose expression seems to be mainly in the in testis, stomach, uterus, neutrophils, thyroid, lung and kidney (Garcia et al. 2001).

A lot of studies indicate that these molecules could play an important role in host defence, having both a direct, broad spectrum but salt sensitive antimicrobial activity (Lehrer and Ganz, 2002), and also playing a role in mobilizing other components of innate or adaptive immunity (Yang et al., 2002).

Twenty eight other putative $\beta$-defensin genes have been identified in the human genome, using a bioinformatic technique, based primarily on the 6-cysteine motif present in these molecules (Schutte et al., 2002).

Schutte et al. (2002) discovered five conserved bdefensin gene clusters in human and analogous clusters in mouse, using a computational search strategy: they identified the putative second exons of 28 new human and 43 new mouse $\beta$-defensin genes organized in five syntenic chromosomal regions. Within each cluster, the similarity of gene sequences and organization suggested each cluster pair arose from a common ancestor and was retained because of conserved functions. Other authors (Semple et 
al., 2003) used a strategy that combined a bioinformatic approach and RT-PCR gene expression analysis to identify new $\beta$-defensin genes. Using a hidden Markov model constructed from the alignment of over 40 mammalian GenBank $\beta$-defensin sequences, they identified four new $\beta$-defensin genes in addition to those coding for the four known molecules, in BAC clone sequences localized to the 8p22-p23 region. These genes were confirmed to be functional by RT-PCR amplification.

In our study we have used a FISH approach based on two BAC clones that contain one or more of the four well-characterized human $\beta$-defensins (DEFB1, DEFB4, DEFB103 and DEFB104) to localize homologous genes on metaphasic chromosomes of the great apes and several non-human primate species, as well as two prosimians, in order to investigate their genetic mapping and to verify the presence of $\beta$-defensin genes in chromosomal regions other than 8p23-p.22 as described by Schutte et al. (2002).

\section{Materials and Methods}

\section{Cell lines}

Fibroblast cells from Pan troglodytes (PTR), Gorilla gorilla (GGO) Pongo pygmaeus (PPY), Macaca mulatta (MMU), Cercopithecus aethiops (CAE), Presbytis cristata (PCR), Callithrix jacchus (CJA), Callicebus moloch (CMO), Lemur catta ( $L C A)$ and Eulemur macaco macaco (EMA) were grown in Dulbecco's minimal essential medium (Gibco-BRL Rockville,MD, USA) supplemented with $10 \%$ fetal calf serum, $2 \mathrm{mM}$ L-glutamine and penicillin-streptomycin sulfate and cultured at $37^{\circ} \mathrm{C}$.

\section{Bac clones}

The BAC clones RP11-791N7, RP11-287p18, and RP11-351d16 were obtained from UCSC database, April 2003. The former two are in the 8p22-p23 region (http://genome.ucsc.edul, April 2003), the latter clone is a test probe containing the conserved RET gene.

\section{FISH experiments}

Chromosome preparations were obtained following standard protocols. For stretched chromosomes, cells were resuspended in hypotonic solution ( $\mathrm{HCM}$ : hepes, $100 \mathrm{mM}$; glicerol, 1 M; $\mathrm{CaCl}_{2}$, $100 \mathrm{mM}$; $\mathrm{MgCl}_{2}$, $0.5 \mathrm{M}$, taken to $10 \mathrm{ml}$ in $\mathrm{H}_{2} \mathrm{O}$ ) and left for $15 \mathrm{~min}$ utes. The resuspension was centrifuged using a cytospin (800-1200 rpm for 5-15 minutes) in order to produce stretched chromosomes (Laan et al., 1995). Slides were hybridized in situ basically as described by Lichter et al. (1990), with minor modifications. BAC probes ( $200 \mathrm{ng}$ ) were labelled by nick translation using nucleotides modified with fluorocrome cy3; hybridization was performed at $37^{\circ} \mathrm{C}$ in 2XSSC, $50 \%(\mathrm{v} / \mathrm{v})$ formamide, $10 \%(\mathrm{v} / \mathrm{v})$ dextran sulfate, $5 \mu \mathrm{g}$ human Cotl DNA (Gibco-BRL), and 3 $\mu \mathrm{g}$ sonicated salmon sperm DNA, in a volume of 10 $\mathrm{ml}$. Post-hybridization washings were at $60^{\circ} \mathrm{C}$ in $0.1 \times$ SSC (three times, high stringency). Washes of FISH experiments using human probes on primates were performed at lower stringency: $37^{\circ} \mathrm{C}$ in $2 \times S S C$ $50 \%$ formamide $(\times 3)$, followed by three washes at $42^{\circ} \mathrm{C}$ in $2 \times \mathrm{SSC}(\times 3)$. Digital images were obtained using a Leica DMRXA epifluorescence microscope equipped with a cooled CCD camera (Princeton Instruments, NJ, USA). DAPI was used to counterstain the chromosomes in order to recognize them on the basis of the DAPI banding pattern. Сy 3 and DAPI fluorescence signals, detected with specific filters, were recorded separately as grey scale images. Pseudocoloring and merging of images were performed using Adobe Photoshop ${ }^{\mathrm{TM}}$ software.

\section{Results and Discussion}

We have performed a query on the UCSC database (http://genome.ucsc.edu/ April 2003) using the mRNA sequences of four known $\beta$-defensins, corresponding to the genes: DEFBI (NM_005218); $D E F B 4$ (formerly DEFB2, NM_004942.2); $D E F B 103$ (formerly DEFB3, NM_018661) and $D E F B 104$ (formerly DEFB4, NM_080389) and found the corresponding BAC clones, which are listed in Table 1. We then used the RP11-287p18 clone, containing the DEFB4-DEFB103-DEFB104 cluster

Table 1. Bac clone localization of defensin genes (position is referred to UCSC database April 2003).

\begin{tabular}{lccc}
\hline \hline Bac clone & Defensin gene & Mapping & Position(kb) \\
\hline RP11-791n7 & DEFB1 & Chr 8 & $6736-6925$ \\
RP11-287p18 & DEFB4-DEFB103-DEFB104 cluster 1 & Chr 8 & $7330-7501$ \\
\hline
\end{tabular}



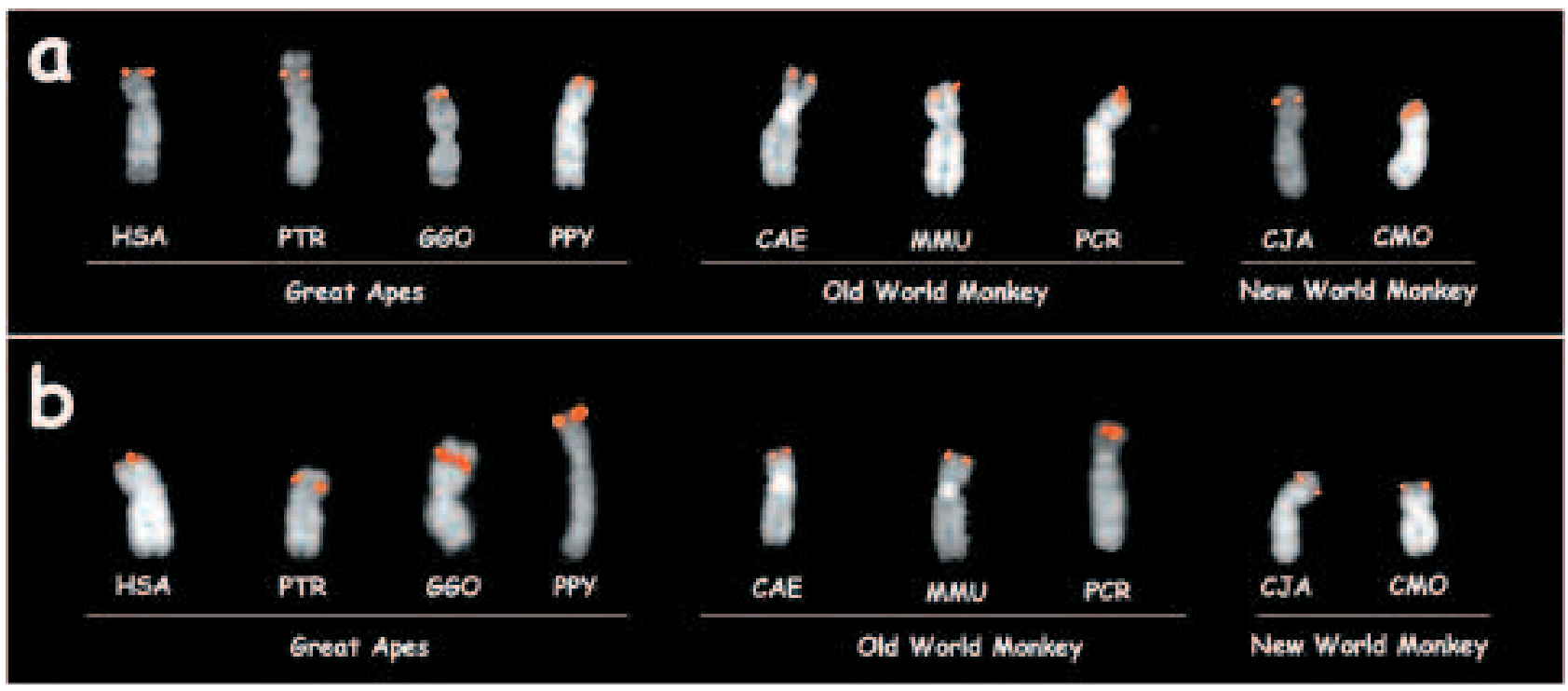

Figure 1. Comparative FISH analysis of the $\beta$-defensin genes. In situ hybridization of the BAC RP11-791n7 probe, containing the DEFB1 gene (a) and of the BAC RP11-287p18 probe, containing the DEFB4DEFB103-DEFB104 cluster (b) onto metaphasic chromosome preparations from Homo saplens (HSA), the Great Apes Pan troglodytes (PTR), Gorlla gorllia (GGO) and Pongo pygmaeus (PPY), the Old World Monkeys Macaca mulatta (MMU), Cercopithecus aethlops (CAE) and Presblt/s crystata (PCR) and the New World Monkeys: Callitrix jacchus (CJA) and Callicebus moloch (CMO). (a) chromosome 8 for HSA, 8 for PTR GGO, PPY, CAE and MMU; 7 for PCR, 13 for CJA and 9 for CMO (b) from left to right, chromosome 8 HSA, 8 PTR GGO and PPY; 8, for CAE; 8 for MMU; 7, for PCR; 13 for CJA and 9 for CMO. Human chromosome 8 is the homologous of the reported non-human primate chromosomes.

and the RPI1-791n7 clone, containing only DEFBI (Table 1) for the comparative mapping of these genes in primates.

Metaphasic chromosomal preparations of ten nonhuman primate species, including Pan troglodytes (PTR), Gorilla gorilla (GGO), Pongo pygmaeus (PPY) (Great Apes group), Macaca mulatta (MMU), Cercopithecus aethiops (CAE), Presbytis cristata (PCR) (Old World Monkeys group), Callithrix jacchus (CJA), Callicebus moloch (CMO) (New World Monkeys group), Lemur catta (LCA) and Eulemur macaco macaco (EMA) (prosimians group) were hybridized with the two BACs. The RP11-791n7 clone (containing DEFB1) showed signals in correspondence to 8p23-p22 in human preparations and in homologous bands in chromosomes from the non-human primate species (Figure 1A), but not in prosimian chromosomes (LCA and EMA, see Figure 2A). In the latter case co-hybridization experiments, using the RP11-351D16 BAC clone, which contains the evolutionarily conserved RET gene, as test probe (Carbone et al. 2002), were performed to confirm the negative FISH results obtained on LCA and EMA chromosomes. A positive hybridization signal was detected only for the test probe (Figure $2 \mathrm{~B}$ ).

The RP11-287p18 BAC clone, containing the
DEFB4, DEFB103, DEFB104 cluster, detected a signal on human chromosome 8 , as well as in homologous regions for the Great Apes. Homologous regions to chromosome 8 were detected in the Old and New world Monkeys (see Figure 1B and Table 2). This clone, like RP11-791n7, did not result in a FISH signal with prosimian metaphasic chromosome preparations (which were positive for the test probe in co-hybridization experiment).

In humans, four $\beta$-defensins have been isolated and well-characterised so far, and their genes (DEFB1, DEFB4, DEFB103 and DEFB104) map on chromosome 8 (8p22-p23). We have identified BAC probes containing these genes and localized homologous regions on different non-human primate chromosomes by using fluorescence in situ hybridization (FISH). The localization of DEFBI (BAC clone RP11-791n7) on this chromosome in the different primate species indicated a strong conservation of the homology ranging from humans to New World Monkeys. The localization of the $D E F B 4, D E F B 103$ and DEFB104 gene cluster (BAC clone RP11-287p18) indicated the presence of these genes on chromosome 8 (or homolog chromosomes) in the Great apes, in the Old World Monkeys and in the New World Monkeys.

The e-cloning results obtained by Schutte et al 

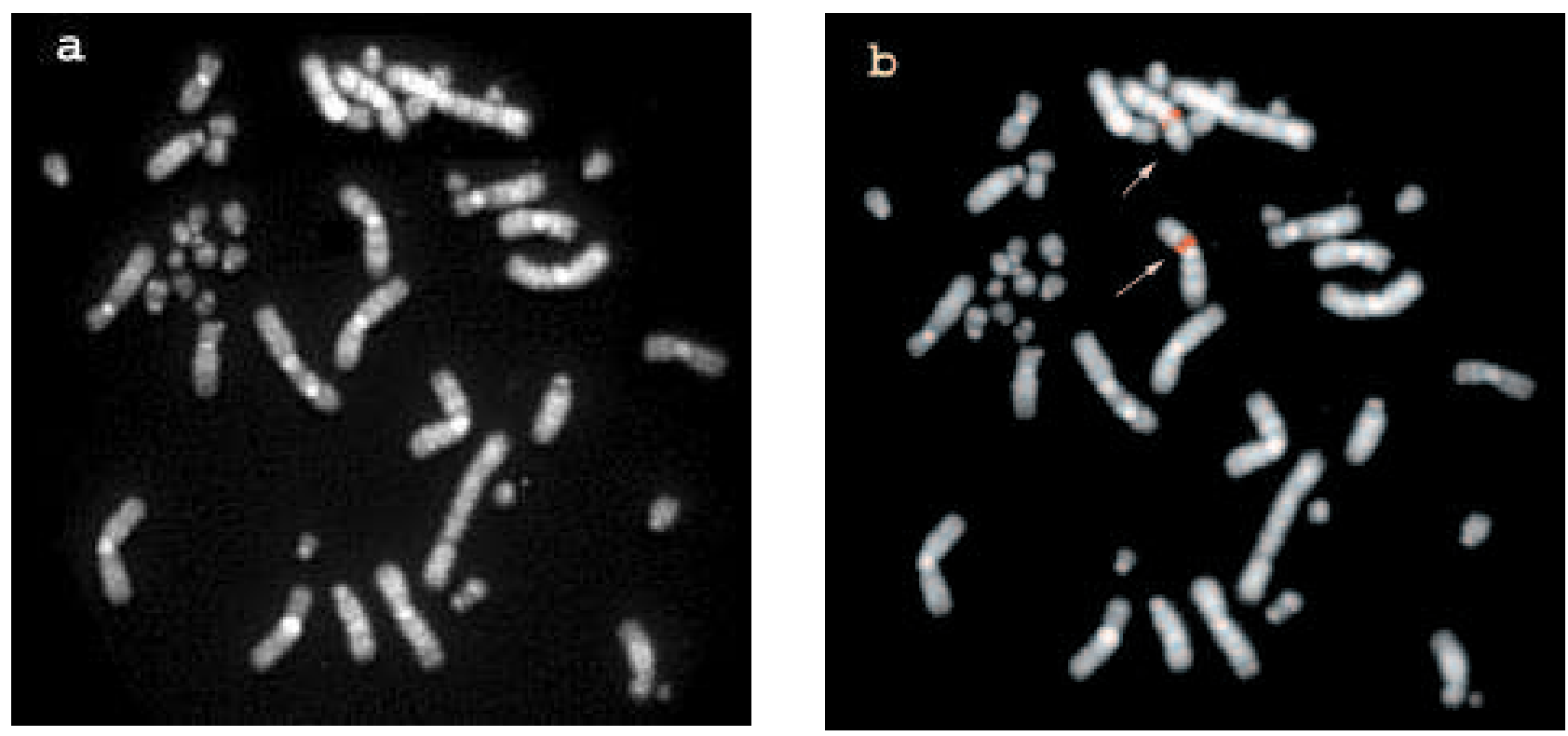

Figure 2. In situ hybridization of the BAC RP11-791n7 probe, containing the DEFB1 gene and of the BAC RP11-351d16, containing the conserved RET gene, onto metaphasic chromosome preparations from E. macaco (EMA). No hybridization was detected for both BAC RP11-791n7 (a). A positive signal of hybridization is visible (arrows) only for BAC RP11-351d16, the control probe (b).

Table 2. Comparative FISH mapping. Mapping on non-human primates was performed following Yunis (1982) nomenclature for Great Apes, Finelli et al. (1999) for Cercop/thecus aethlops (CAE), Wienberg et al. (1992) for Macaca mulatta (MMU), Bigoni et al. (1997) for Presbyt/s cristata (PCR), Sherlock et al. (1996) for Callitrix facchus (CJA) and Stanyon ot al. (2000) for Callicebus moloch (CM0). In each species signals are localized on homologues region of human chromosome 8.

\begin{tabular}{|c|c|c|c|c|c|c|c|c|c|}
\hline$B A C$ & $H S A$ & PTR & GGO & PPY & CAE & MMU & $P C R$ & CJA & CMO \\
\hline RP11-791n7 & 8 & 8 & 8 & 8 & 8 & 8 & 7 & 13 & 9 \\
\hline RP11-287p18 & 8 & 8 & 8 & 8 & 8 & 8 & 7 & 13 & 9 \\
\hline
\end{tabular}

(2002) using a bioinformatic approach, indicate the existence of three other $\beta$-defensin gene clusters localized in humans on chromosomes $6 \mathrm{pl2}$, $20 \mathrm{ql} 1.1$ and 20pl. In our study no other putative $\beta$-defensin genes were found by FISH in these regions in the human or monkey genomes.

This discrepancy could derive from the different approaches used to study the localization and synteny of $\beta$-defensin. Schutte et al. (2002) method to detect defensin genes in different chromosomal regions was based on the hypothesis that all $\beta$ defensins should share the characteristic six-cystein motif, and thus concerned only exon 2. However, their data are not supported by experimental verification of gene expression and functionality. Our FISH approach has led us to identify chromosomal regions with $\beta$-defensin genes, in both human and non-human primate chromosomes, that shared similarity at the level of their whole nucleotide sequence.

\section{Acknowledgments}

CEGBA (Centro di Eccellenza Geni in campo Biosanitario e Agroalimentare) and European Commission (INPRIMAT, QLRI-CT-2002-01325) are gratefully acknowledged for financial support.

\section{Funding}

This work was partially supported by a grant COFIN 2001. M. Boniotto is recipent of a long term fellowship from the University of Trieste, Italy.

\section{References}

Bensch KW, Raida M, Magert HJ, Schulz-Knappe P, Forssmann WG. hBD-1: a novel $\beta$-defensin from human plasma. FEBS Lett 1995;368:331-5.

Bigoni F, Stanyon R, Koehler U, Morescalchi AM, Wienberg J. Mapping homology between human and black and white colobine monkey chromosomes by fluorescent in situ hybridization. Am J Primatol 1997;42:289-98.

Carbone L, Ventura M, Tempesta S, Rocchi M, Archidiacono N. Evolutionary history of chromosome 10 in primates. Chromosoma 
2002;111: 267-72.

Diamond G, Russell JP, Bevins CL. Inducible expression of an antibiotic peptide gene in lipopolysaccharide-challenged tracheal epithelial cells. Proc Natl Acad Sci USA;1996;93:5156-60.

Finelli $\mathrm{P}$, Stanyon R, Plesker R, Ferguson-Smith MA, O'Brien PC, Wienberg $\mathrm{J}$. Reciprocal chromosome painting shows that the great difference in diploid number between human and African green monkey is mostly due to non-Robertsonian fissions. Mamm Genome 1999;10:713-8.

Garcia JR, Krause A, Schulz S, Rodriguez-Jimenez FJ, Kluver E, Adermann $\mathrm{K}$, et al. Human $\beta$-defensin 4: a novel inducible peptide with a specific salt-sensitive spectrum of antimicrobial activity. Faseb J 2001;15:1819-21.

Goldman MJ, Anderson GM, Stolzenberg ED, Kari UP, Zasloff M, Wilson, JM. Human $\beta$-defensin- 1 is a salt-sensitive antibiotic in lung that is inactivated in cystic fibrosis. Cell 1997 88:553-60.

Harder J, Bartels J, Christophers E, Schroder JM. A peptide antibiotic from human skin. Nature 1997;387:861.

Harder J, Siebert R, Zhang $Y$, Matthiesen P, Christophers E, Schlegelberger $B$, et al. Mapping of the gene encoding human $\beta$-defensin2 (DEFB2) to chromosome region 8p22-p23.1. Genomics $1997 ; 46: 472-5$

Hiratsuka T, Nakazato M, Date Y, Ashitani J, Minematsu T, Chino N, et al. Identification of human $\beta$-defensin-2 in respiratory tract and plasma and its increase in bacterial pneumonia. Biochem Biophys Res Commun 1998;249:943-7.

Laan M, Kallioniemi OP, Hellsten E, Alitalo K, Peltonen L, Palotie A. Mechanically stretched chromosomes as targets for high-resolution FISH mapping. PCR Methods Appl 1995;5:13-20.

Lehrer RI, Ganz T. Defensins of vertebrate animals. Curr Opin Immunol 2002;14:96-102.

Lichter $\mathrm{P}$, Ward DC. Is non-isotopic in situ hybridization finally coming of age? Nature 1990;345:93-5.

Liu L, Wang L, Jia HP, Zhao C, Heng HH, Schutte BC, et al. Structure and mapping of the human $\beta$-defensin HBD-2 gene and its expression at sites of inflammation. Gene 1998;222:237-44.

Mathews M, Jia HP, Guthmiller JM, Losh G, Graham S, Johnson GK, et al. Production of $\beta$-defensin antimicrobial peptides by the oral mucosa and salivary glands. Infect Immun 1999;67:2740-5.

Schonwetter BS, Stolzenberg ED, Zasloff MA. Epithelial antibiotics induced at sites of inflammation. Science 1995;267:1645-8.

Schutte BC, Mitros JP, Bartlett JA, Walters JD, Jia HP, Welsh MJ, et al. Discovery of five conserved $\beta$-defensin gene clusters using a computational search strategy. Proc Natl Acad Sci USA 2002;99: 2129-33.

Semple CA, Rolfe M, Dorin JR. Duplication and selection in the evolution of primate $\beta$-defensin genes. Genome Biol 2003; R31:4.

Sherlock JK, Griffin DK, Delanthy JDA, Parrington JM. Homologies between human and marmoset (Callitrix Jacchus) chromosomes revealed by comparative chromosome painting. Genomics 1996;33: 214-9.

Stanyon R, Consigliere S, Muller S, Morescalchi A, Neusser M, Wienberg J. Fluorescence in situ hybridization (FISH) maps chromosomal homologies between the dusky titi and squirrel monkey. Am J Primatol 2000;50:95-107.

Wienberg J, Stanyon R, Jauch A, Cremer T. Homologies in human and Macaca fuscata chromosomes revealed by in situ suppression hybridization with human chromosome specific libraries. Chromosoma 1992;101:265-70.

Yang D, Biragyn A, Kwak LW, Oppenheim JJ. Mammalian defensins in immunity: more than just microbicidal. Trends Immunol 2002; 23:291-6.

Yunis JJ, Prakash 0. The origin of man: a chromosomal pictorial legacy. Science 1982;215:1525-30.

Zhao C, Wang I, Lehrer, RI. Widespread expression of $\beta$-defensin hBD1 in human secretory glands and epithelial cells. FEBS Lett 1996;396:319-22. 
M. Ventura et al. 